\title{
EVERSHED EFFECT IN SUISSPOTS AND THEIR CANOPIES
}

\section{S.K. SOLANKI \& C. MONTAVON}

Institute of Astronomy, ETH-Zentrum, CH-8092 Zürich, Switzerland

\section{W. LIVINGSTON}

National Solar Observatory, NOAO^, P.O.Box 26732, Tucson AZ 85726, USA

\begin{abstract}
An analysis of Stokes $I$ and $V$ profiles of $1.56 \mu \mathrm{m}$ lines of sunspots near the solar limb shows that the magnetic field continues outside the visible contours of sunspots in the form of a low-lying superpenumbral canopy. We also find that the $V$ profiles formed in the canopy exhibit the Evershed effect (with line shifts of $1-2 \mathrm{~km} \mathrm{~s}^{-1}$ ), while the matter below it shows no sign of a flow. Therefore, the Evershed effect definitely is present beyond the visible sunspot boundary. However, if we interpret the line shifts in terms of stationary flows, then only small fraction of the matter seen to be flowing outwards in the penumbra can be accounted for by the outward flow in the superpenumbral canopy. Therefore, although the Evershed "flow" does not stop at the boundary of the spot, most of the flowing matter stops or disappears there.
\end{abstract}

Keywords: Solar magnetic fields - Sunspots - Evershed flow

\section{INTRODUCTION}

Some investigators claim that the Evershed effect ends sharply at the penumbral boundary (e.g. Wiehr \& Degenhardt 1992), while others claim that it continues beyond the visible sunspot boundary (e.g. Börner \& Kneer 1992). To resolve this inconsistency, we analyse Stokes $I$ and $V$ spectra of the Landé $g=3, \mathrm{Fe}$ I $1.5648 \mu \mathrm{m}$ and $g_{e f f}=1.53$, Fe I $1.5653 \mu$ mlines. Due to the extremely high magnetic sensitivity of the $g=3$ line ( 3 times larger than of lines in the visible), it is possible to follow the magnetic field and search for the associated Evershed effect beyond the visible sunspot.

\section{DATA AND ANALYSIS}

Stokes $I$ \& $V$ profiles were obtained with the McMath telescope, vertical spectrograph and Baboquivari detector (Livingston 1991) along a slice through a sunspot observed near the solar limb $(\mu=\cos \vartheta \approx 0.5)$. We determine magnetic

* Operated by the Association of Universities for Research in Astronomy, Inc. (AURA) under cooperative agreement with the National Science Foundation. 
field strength and inclination, velocity shift and broadening, stray-light and temperature from these data by inverting them using synthetic profiles that are numerical solutions of the Unno-Rachkovski equations in realistic model atmospheres (see Solanki et al. 1992 for more on the technique).

\section{RESULTS}

The Stokes $I$ profiles in the penumbra are highly asymmetric and shifted, suggestive of the Evershed effect. Fig. 1 shows a Stokes $I$ and $V$ spectrum each from the discward (solid) and limbward (dashed) penumbra of a spot. A simple model of a stationary flow, with a velocity of approximately $2.5-4 \mathrm{~km}$ $\mathrm{s}^{-1}$ at $\log \tau=-1$ (close to the formation height of the lines) can simultaneously reproduce the $I$ and $V$ profiles of both lines.

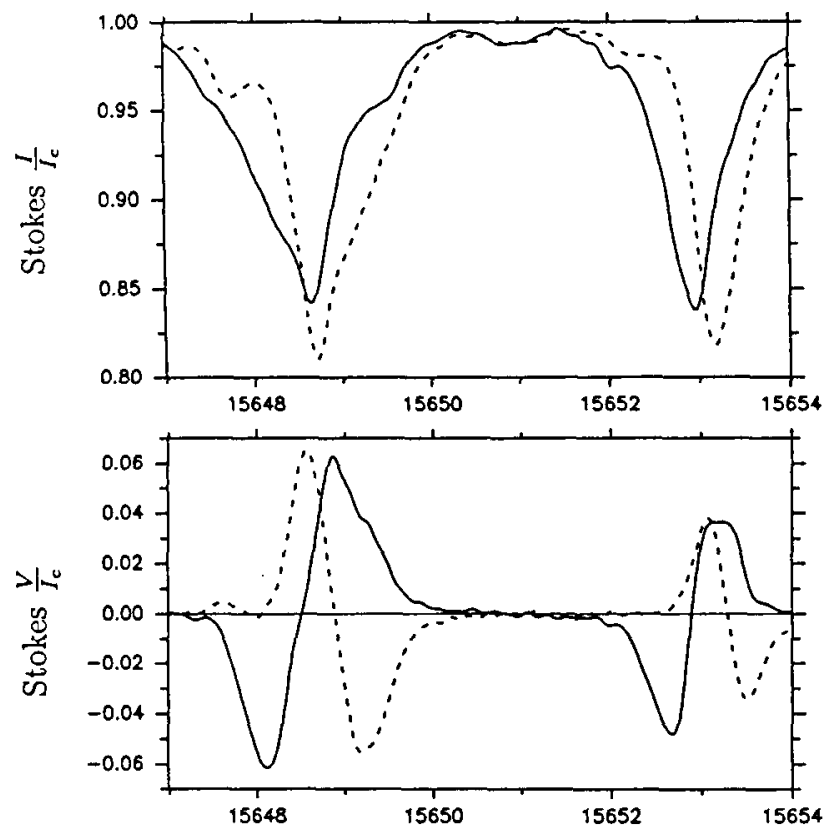

Wavelength $\lambda[\AA]$

Fig. 1. Profiles observed in the penumbra. a) Stokes $I$, b) Stokes $V$.

$V$ profiles are also present close to, but outside the visible sunspots. Such profiles are best interpreted in terms of a low-lying magnetic canopy, i.e. an almost horizontal magnetic field overlying a relatively undisturbed, nonmagnetic atmosphere (Giovanelli \& Jones 1982, Solanki et al. 1992). The height 


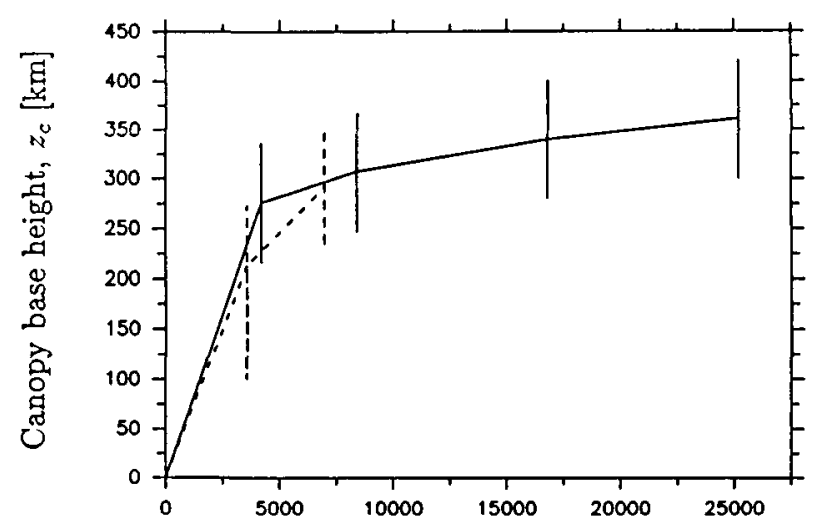

Distance from penumbra edge $r-r_{p}[\mathrm{~km}]$

Fig. 2. Base height of the superpenumbral canopy, $z_{c}$, vs. distance from the outer penumbral boundary, $r-r_{p}$. Solid line: $z_{c}$ on the limbward side of the sunspot, dashed line: $z_{c}$ on the discward side.

of the canopy base determined from the present data is plotted in Fig. 2. It agrees well with previous determinations.

The $V$ profiles, which are formed exclusively above the canopy base, are shifted in wavelength relative to the Stokes $I$ profiles, which are formed mainly below the canopy. In Fig. 3a (resp. 3b) we plot the $V$ and $I$ profiles observed in the discward superpenumbra of the sunspot. The figure clearly shows that the $V$ profile is blueshifted relative to the $I$ profile. Similarly, in the limbward superpenumbra $V$ is redshifted. The observed Stokes $V$ shifts correspond to an outflow of $1-2 \mathrm{~km} \mathrm{~s}^{-1}$ in the canopy, but no systematic in or outflow $>0.5 \mathrm{~km}$ $\mathrm{s}^{-1}$ is seen below the canopy in Stokes $I$.

\section{DISCUSSION AND CONCLUSIONS}

Our results show clearly that the Evershed flow extends beyond the sunspot boundary. They also indicate the reason for the discrepancy between previous investigations which showed an Evershed flow extending beyond the sunspot boundary (e.g. Küveler \& Wiehr 1985, Dialetis et al. 1985, Alissandrakis et al. 1988, Börner \& Kneer 1992) and those showing an abrupt stop at the boundary (e.g. Wiehr et al. 1986, Wiehr \& Balthasar 1989, Title et al. 1992, Wiehr \& Degenhardt 1992). All previous measurements were carried out using Stokes $I$ alone, which is ill suited to decide this question. For example, if a line with a low formation height is used, it will detect no flow outside the sunspot (since the flow is present only above the canopy base), while a line formed at greater photospheric heights will detect a flow.

If the Evershed effect is due to a mass flow, then the mass flux of the flow in the canopy is only a small fraction of the mass flux observed in the penumbra. Recall that the $1.56 \mu \mathrm{m}$ line see a velocity near $\tau_{c}=1$ in the penumbra. At this height the gas density is $\approx 2.9 \cdot 10^{-7} \mathrm{~g} \mathrm{~cm}^{-3}$. In the superpenumbral canopy 


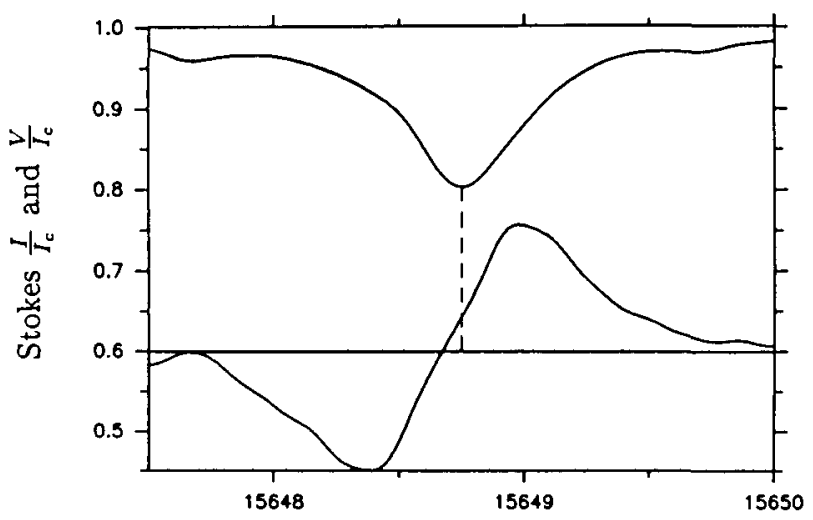

Wavelength $\lambda[\AA]$

Fig. 3. Stokes $I$ and $V$ profiles observed in the discward side of the superpenumbral canopy of the sunspot. The $V$ profiles have been vertically shifted and scaled.

(approximately $5000 \mathrm{~km}$ outside the spot), on the other hand, the observed velocity is smaller and is only present at $z>250-300 \mathrm{~km}$ (c.f. Fig. 3), at which height the gas density is $\approx 5.7 \cdot 10^{-8} \mathrm{~g} \mathrm{~cm}^{-3}$. Even allowing the field to expand in cross-section with radius, we can still say that, as a conservative estimate, less than $20-30 \%$ of the total mass transported by the Evershed flow inside the penumbra appears to reach the canopy.

Therefore, we conclude that the Evershed effect persists outside sunspots, but that, if the observed line shifts are due to a mass flow, most of the mass must be deposited within the visible outlines of the sunspot.

\section{REFERENCES}

Alissandrakis C.E., Dialetis D., Mein P., Schmieder B., Simon G., 1988, Astron. Astrophys. 201, 339

Börner P., Kneer F., 1992, Astron. Astrophys. 255, 307

Dialetis D., Mein P., Alissandrakis C.E., 1985, Astron. Astrophys. 147, 93

Giovanelli, R.G., Jones, H.P., 1982, Solar Phys. 79, 267

Küveler G., Wiehr E., 1985, Astron. Astrophys. 142205

Livingston W., 1991, in Solar Polarimetry, L. November (Ed.), National Solar Obs., Sunspot, NM, p. 356

Solanki S.K., Rüedi I., Livingston W., 1992, Astron. Astrophys. 263, 339

Title A.M., Frank Z.A., Shine R.A., Tarbell T.D., Topka K.P., Scharmer G., Schmidt W., 1992, in Sunspots: Theory and Observations, J.H. Thomas, N.O. Weiss (Eds.), Kluwer, Dordrecht, p. 195

Wiehr E., Balthasar H., 1989, Astron. Astrophys. 208, 303

Wiehr E., Degenhardt D., 1992, Astron. Astrophys. 259, 313

Wiehr E., Stellmacher G., Knölker M., Grosser H., 1986, Astron. Astrophys. 155, 402 